03

\title{
Отражение света слоем гиперболического метаматериала в случае распространения в нем особых неоднородных волн
}

\author{
(C) Н.С. Петров ${ }^{1}$, С.Н. Курилкина ${ }^{2}$, А.Б. Зимин ${ }^{3}$, В.Н. Белый ${ }^{2}$ \\ ${ }^{1}$ Институт повышения квалификации по новым направлениям развития техники, \\ технологий и экономики \\ Белорусского национального технического университета, \\ 220107 Минск, Беларусь \\ ${ }^{2}$ Институт ффизики им. Б.И. Степанова НАН Беларуси, \\ 220072 Минск, Беларусь \\ ${ }^{3}$ Белорусский государственный университет информатики и радиоэлектроники, \\ 220013 Минск, Беларусь \\ e-mail: s.kurilkina@ifanbel.bas-net.by
}

Поступила в редакцию 26.10.2018 г.

В окончательной редакции 26.10.2018 г.

Принята к публикации 06.11.2018 г.

\begin{abstract}
Приведено решение граничной задачи об отражении электромагнитных волн слоем гиперболического метаматериала в условиях полного отражения на границе с ним и отсутствии явления двупреломления. В этом случае в нем возможно распространение так называемых особых неоднородных электромагнитных волн. Получены аналитические выражения для векторных амплитуд отраженных и прошедших слой волн, а также для распространяющихся в нем особых неоднородных электромагнитных волн, позволяющие рассчитать энергетические характеристики отраженного и прошедшего излучения для случая оптически одноосного метаматериала.
\end{abstract}

DOI: $10.21883 /$ OS.2019.03.47373.311-18

\section{Введение}

В течение последнего десятилетия появилось большое число публикаций, посвященных исследованию метаматериалов, проявляющих электромагнитные свойства, не характерные для обычных изотропных и анизотропных сред [1,2]. Это обусловлено перспективами их использования для управления световыми пучками, в литографии, для получения изображений с субволновым разрешением [3,4]. Такие среды могут быть описаны усредненными (эффективными) проницаемостями (диэлектрической $\varepsilon$ и магнитной $\mu$ ), отличающимися от таковых для образующих их материалов [5]. Эффективные проницаемости метаматериалов существенно зависят от геометрических размеров нановключений и их взаимного расположения. Таким образом, путем изменения данных параметров можно получать электромагнитный отклик среды и, следовательно, достигать эффективных значений $\varepsilon$ и $\mu$, невозможных для естественных анизотропных материалов.

Одним из видов метаматериалов являются гиперболические метаматериалы (ГММ), для которых предполагается $\mu \approx 1$, а описывающий их диагональный тензор диэлектрической проницаемости $\varepsilon=\operatorname{diag}\left\{\varepsilon_{o}, \varepsilon_{o}, \varepsilon_{e}\right\}$ имеет главные значения проницаемостей (поперечной $\varepsilon_{o}$ и продольной $\varepsilon_{e}$ ), различающиеся знаком [6]. Это обусловливает появление гиперболической (а не эл- липтической, наблюдаемой у обычных диэлектриков) дисперсии.

Ряд важных практических применений метаматериалов (в частности, в сенсорике, ближнепольной микроскопии, в системах получения изображений) обусловлен особенностями формируемых в них электромагнитных волн. Среди них особое место занимают неоднородные волны, у которых плоскости равных фаз и равных амплитуд не параллельны между собой. Такие волны возникают, например, в прозрачных средах при полном отражении света, а также при наклонном падении излучения в поглощающих (усиливающих) материалах. В монографии [7] показана возможность существования неоднородных волн особого вида, удовлетворяющих уравнениям Максвелла, векторная амплитуда которых изменяется с глубиной проникновения волны в кристалл не по экспоненциальному, а более сложному закону. Такие особые неоднородные волны возникают, в частности, при полном отражении в оптически одноосном прозрачном кристалле при отсутствии в нем явления двулучепреломления [8]. В работе [9] показана возможность распространения подобных неоднородных волн особого типа на границе гиперболического метаматериала. В настоящей работе рассмотрен более реальный случай, а именно отражение света анизотропным слоем метаматериала. 


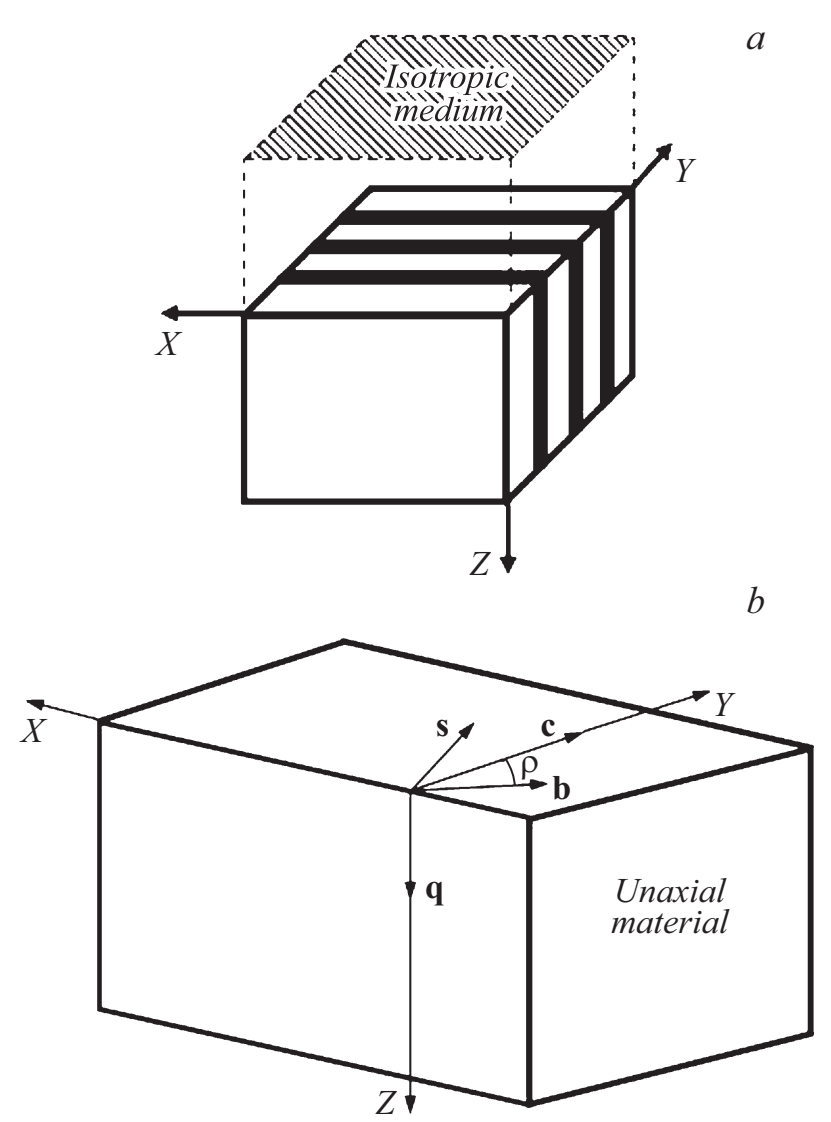

Рис. 1. Схема рассматриваемой структуры $(a)$ и ее представление в приближении эффективной среды $(b)$. c - единичный вектор вдоль оптической оси, $\mathbf{b}, \mathbf{q}, \mathbf{s}-$ тройка единичных векторов, где q - вектор, ортогональный границе раздела, $(\mathbf{c}, \mathbf{q})$ - главная плоскость анизотропной среды, $(\mathbf{b}, \mathbf{q})-$ плоскость падения световой волны, повернутая относительно главной плоскости на угол $\rho$.

\section{Особенности отражения света слоем гиперболического метаматериала в случае распространения в нем неоднородных волн особого типа}

Пусть изотропная среда с диэлектрической проницаемостью $\varepsilon_{1}$ граничит со слоем гиперболического метаматериала, характеризуемого тензором диэлектрической проницаемости $\varepsilon=\operatorname{diag}\left\{\varepsilon_{o}, \varepsilon_{o}, \varepsilon_{e}\right\}=\varepsilon_{o}+\delta \mathbf{c} \cdot \mathbf{c}$, где $\delta=\varepsilon_{e}-\varepsilon_{o}, \mathbf{c}-$ единичный вектор вдоль оптической оси (точка между векторами обозначает их диадное произведение). В дальнейшем будем использовать систему координат, ось $z$ которой направлена внутрь анизотропной среды, а ее начало совпадает с входной гранью слоя (рис. 1).

Пусть на границу раздела изотропного диэлектрика и слоя ГММ падает однородная линейно поляризованная электромагнитная волна в условиях полного отражения при отсутствии в анизотропной среде явления двупреломления.
При решении поставленной задачи будем исходить из уравнений Максвелла

$$
\operatorname{rot} \mathbf{E}+\frac{1}{c} \frac{\partial \mathbf{B}}{\partial t}=0, \quad \operatorname{rot} \mathbf{H}-\frac{1}{c} \frac{\partial \mathbf{D}}{\partial t}=0,
$$

где $\mathbf{D}=\varepsilon \mathbf{E}, \mathbf{B}=\mu \mathbf{H}(\mu=1)$, т. е. будем считать среды немагнитными.

Представим векторы напряженности электрического поля падающей из изотропного диэлектрика $\left(\mathbf{E}_{1}\right)$ и отраженной $\left(\mathbf{E}_{1}^{\prime}\right)$ анизотропным слоем плоских волн, гармонически зависящих от времени $\left(\sim e^{-i \omega t}\right)$, в следующем виде:

$$
\begin{array}{r}
\mathbf{E}_{1}=\left(A_{1} \mathbf{s}+B_{1}\left[\mathbf{n}_{1} \mathbf{s}\right]\right) \exp \left(i \varphi_{1}\right), \\
\left.\mathbf{E}_{1}^{\prime}=\left(A_{1}^{\prime} \mathbf{s}+B_{1}^{\prime}\left[\mathbf{n}_{1}^{\prime} \mathbf{s}\right]\right)\right) \exp \left(i \varphi_{1}^{\prime}\right) .
\end{array}
$$

Здесь $A_{1}\left(B_{1}\right)$ и $A_{1}^{\prime}\left(B_{1}^{\prime}\right)$ - амплитуды волн с векторами поляризации, ориентированными соответственно перпендикулярно и параллельно плоскости падения, $\varphi_{1}=k \mathbf{m}_{1} \mathbf{r}, \varphi_{1}^{\prime}=k \mathbf{m}_{1}^{\prime} \mathbf{r}(\mathbf{r}-$ радиус-вектор),$k-$ волновое число в вакууме $\mathbf{m}_{1}$ и $\mathbf{m}_{1}^{\prime}$ - так называемые векторы рефракции этих волн [2], которые можно представить в виде

$$
\mathbf{m}_{1}=n_{1} \mathbf{n}_{1}=\xi \mathbf{b}+\eta_{1} \mathbf{q}, \quad \mathbf{m}_{1}^{\prime}=n_{1} \mathbf{n}_{1}^{\prime}=\xi \mathbf{b}-\eta_{1} \mathbf{q},
$$

причем для них имеют место соотношения

$$
\mathbf{m}_{1}^{2}=\left(\mathbf{m}_{1}^{\prime}\right)^{2}=n_{1}^{2}=\varepsilon_{1}=\xi^{2}+\eta_{1}^{2},
$$

где $n_{1}=\sqrt{\varepsilon_{1}}-$ показатель преломления изотропной среды, из которой падает излучение, $\mathbf{n}_{1}$ и $\mathbf{n}_{1}^{\prime}-$ единичные векторы в направлении распространения фазы волны, $\xi=n_{1} \sin \alpha, \eta_{1}=n_{1} \cos \alpha, \alpha-$ угол, изменяющийся в интервале $\alpha_{0} \leq \alpha \leq \pi / 2\left(\varepsilon_{0} \leq \xi^{2} \leq \varepsilon_{1}\right)$, $\mathbf{b}$, $\mathbf{q}$ и $\mathbf{s}-$ тройка взаимно перпендикулярных ортов (bвдоль границы раздела сред, q - нормаль к границе анизотропной среды, направленная вглубь нее, s нормаль к плоскости падения $(\mathbf{s}=[\mathbf{b q}])($ рис. $1, b))$.

Векторы напряженности магнитного поля Н также определяются из уравнений (1), откуда для случая плоских волн следует $\mathbf{H}=[\mathbf{m E}]$. Тогда

$$
\begin{aligned}
& \mathbf{H}_{1}=\left(A_{1}\left[\mathbf{m}_{1} \mathbf{s}\right]-n_{1} B_{1} \mathbf{s}\right) \exp \left(i \varphi_{1}\right), \\
& \mathbf{H}_{1}^{\prime}=\left(A_{1}^{\prime}\left[\mathbf{m}_{1}^{\prime} \mathbf{s}\right]-n_{1} B_{1}^{\prime} \mathbf{s}\right) \exp \left(i \varphi_{1}^{\prime}\right) .
\end{aligned}
$$

Для распространяющихся в анизотропном слое особых неоднородных волн воспользуемся соответствующими выражениями для векторов напряженности электрического и магнитного полей волны, приведенными в [9]:

$$
\mathbf{E}=\mathbf{E}_{0} \exp \left(i k \mathbf{m}_{0} \mathbf{r}\right), \quad \mathbf{H}=\mathbf{H}_{0} \exp \left(i k \mathbf{m}_{0} \mathbf{r}\right),
$$

где $\mathbf{m}_{0}=\xi \mathbf{b}+i \gamma \mathbf{q}, \gamma=\sqrt{\xi^{2}-\mathbf{m}_{0}^{2}},\left(\mathbf{m}_{0}^{2}=\varepsilon_{0}\right)$. Здесь в наших обозначениях

$$
\mathbf{E}_{0}=C_{1} \mathbf{N}+C_{2}\left(\mathbf{d}+\delta \xi^{\prime} \mathbf{N}\right),
$$




$$
\mathbf{H}_{0}=\frac{1}{i k} \operatorname{rot} \mathbf{E}_{0}=C_{1}\left[\mathbf{m}_{0} \mathbf{N}\right]+C_{2}\left(\mathbf{d}^{\prime}+\delta \xi^{\prime}\left[\mathbf{m}_{0} \mathbf{N}\right]\right),
$$

где

$$
\begin{gathered}
\mathbf{N}=\left[\mathbf{m}_{0} \mathbf{c}\right], \quad \mathbf{d}=\delta \mathbf{q}-2 \xi\left[\mathbf{m}_{0} \mathbf{s}\right], \\
\mathbf{d}^{\prime}=\left[\mathbf{m}_{0} \mathbf{d}\right]-\delta \gamma \mathbf{c}, \quad \mathbf{c}=\left(\sqrt{\varepsilon_{0}} \mathbf{b}+\gamma \mathbf{s}\right) / \xi, \\
\delta=\varepsilon_{e}-\varepsilon_{0}, \quad \xi^{\prime}=k \mathbf{q r}=k z .
\end{gathered}
$$

Аналогично для отраженных от второй (выходной) границы анизотропного слоя особых волн имеем

$$
\mathbf{E}^{\prime}=\mathbf{E}_{0}^{\prime} \exp \left(i k \mathbf{m}_{0}^{\prime} \mathbf{r}\right), \quad \mathbf{H}^{\prime}=\mathbf{H}_{0} \exp \left(i k \mathbf{m}_{0}^{\prime} \mathbf{r}\right)
$$

где

$$
\begin{gathered}
\mathbf{E}_{0}^{\prime}=C_{1}^{\prime} \mathbf{N}_{1}+C_{2}^{\prime}\left(-\mathbf{d}_{1}+\delta \xi^{\prime} \mathbf{N}_{1}\right), \\
\mathbf{H}_{0}^{\prime}=C_{1}^{\prime}\left[\mathbf{m}_{0}^{\prime} \mathbf{N}_{1}\right]+C_{2}^{\prime}\left(-\mathbf{d}_{1}^{\prime}+\delta \xi^{\prime}\left[\mathbf{m}_{0}^{\prime} \mathbf{N}_{1}\right]\right) .
\end{gathered}
$$

Здесь

$$
\begin{gathered}
\mathbf{N}_{1}=\left[\mathbf{m}_{0}^{\prime} \mathbf{c}\right], \quad \mathbf{d}_{1}=\delta \mathbf{q}-2 \xi\left[\mathbf{m}_{0}^{\prime} \mathbf{s}\right], \quad \mathbf{d}_{1}^{\prime}=\left[\mathbf{m}_{0}^{\prime} \mathbf{d}_{1}\right]+\delta \gamma c, \\
\mathbf{m}_{0}^{\prime}=\xi \mathbf{b}-i \gamma \mathbf{q}, \quad\left(\mathbf{m}_{0}^{\prime 2}=\varepsilon_{0}\right),
\end{gathered}
$$

Векторы электрического и магнитного полей волны, прошедшей анизотропный слой, представим в виде, аналогичном выражениям для $\mathbf{E}_{1}\left(\mathbf{E}_{1}^{\prime}\right)$ и $\mathbf{H}_{1}\left(\mathbf{H}_{1}^{\prime}\right)$, а именно

$$
\begin{gathered}
\mathbf{E}_{2}=\left(A_{2} \mathbf{s}+B_{2}\left[\mathbf{n}_{2} \mathbf{s}\right]\right) \exp \left(i k \mathbf{m}_{2} \mathbf{r}\right), \\
\mathbf{H}_{2}=\left(A_{2}\left[\mathbf{m}_{2} \mathbf{s}\right]-n_{2} B_{2} \mathbf{s}\right) \exp \left(i k \mathbf{m}_{2} \mathbf{r}\right),
\end{gathered}
$$

где $\mathbf{m}_{2}=\xi \mathbf{b}+\eta_{2} \mathbf{q}, \eta_{2}=\sqrt{\mathbf{m}_{2}^{2}-\xi^{2}},\left(\mathbf{m}_{2}^{2}=\varepsilon_{2}=n_{2}^{2}\right), n_{2}-$ показатель преломления изотропной среды, граничащей с анизотропным слоем с другой его стороны.

Используя стандартные граничные условия, можно получить соотношения для амплитуд всех указанных выше волн. В нашем случае граничные условия имеют вид [10]: для первой границы $\left(\xi^{\prime}=k z=0\right)$

$$
\left[\mathbf{E}_{1}+\mathbf{E}_{1}^{\prime}-\mathbf{E}-\mathbf{E}^{\prime}, \mathbf{q}\right]=0, \quad \mathbf{H}_{1}+\mathbf{H}_{1}^{\prime}-\mathbf{H}-\mathbf{H}^{\prime}=0
$$

и для второй границы $\left(\xi^{\prime}=k z=k h\right)$

$$
\left[\mathbf{E}+\mathbf{E}^{\prime}-\mathbf{E}_{2}, \mathbf{q}\right]=0, \quad \mathbf{H}+\mathbf{H}^{\prime}-\mathbf{H}_{2}=0 .
$$

Подставляя в эти векторные равенства выражения (2), (4)-(8) для соответствующих волн, после выполнения определенных вычислительных процедур, получим систему уравнений для нахождения восьми скалярных амплитуд $A_{1}^{\prime}\left(B_{1}^{\prime}\right), C_{1}\left(C_{1}^{\prime}\right), C_{2}\left(C_{2}^{\prime}\right), A_{2}, B_{2}$, т. е. систему с восемью неизвестными, а именно:

$$
\begin{gathered}
A_{1} \mathbf{b}\left[\mathbf{m}_{1} \mathbf{s}\right]+A_{1}^{\prime} \mathbf{b}\left[\mathbf{m}_{1}^{\prime} \mathbf{s}\right]-\mathbf{b} \mathbf{H}_{0}-\mathbf{b} \mathbf{H}_{0}^{\prime}=0, \\
A_{1} \mathbf{q}\left[\mathbf{m}_{1} \mathbf{s}\right]+A_{1}^{\prime} \mathbf{q}\left[\mathbf{m}_{1}^{\prime} \mathbf{s}\right]-\mathbf{q} \mathbf{H}_{0}-\mathbf{q} \mathbf{H}_{0}^{\prime}=0, \\
B_{1} \mathbf{b}\left[\mathbf{n}_{1} \mathbf{s}\right]+B_{1}^{\prime} \mathbf{b}\left[\mathbf{n}_{1}^{\prime} \mathbf{s}\right]-\mathbf{b} \mathbf{E}_{0}-\mathbf{b} \mathbf{E}_{0}^{\prime}=0, \\
n_{1} B_{1}+n_{1} B_{1}^{\prime}+\mathbf{s} \mathbf{H}_{0}+\mathbf{s} \mathbf{H}_{0}^{\prime}=0,
\end{gathered}
$$

$A_{2} \mathbf{b}\left[\mathbf{m}_{2} \mathbf{s}\right] \exp (i \psi)-\mathbf{b H}_{0} \exp (-\vartheta)-\mathbf{b H}_{0}^{\prime} \exp (\vartheta)=0$,

$A_{2} \mathbf{q}\left[\mathbf{m}_{2} \mathbf{s}\right] \exp (i \psi)-\mathbf{q H}_{0} \exp (-\vartheta)-\mathbf{q} \mathbf{H}_{0}^{\prime} \exp (\vartheta)=0$,

$$
\begin{gathered}
\eta_{1} B_{2} \exp (i \psi)-n_{1} \mathbf{b} \mathbf{E}_{0} \exp (-\vartheta)-\mathbf{n}_{1} \mathbf{b} \mathbf{E}_{0}^{\prime} \exp (\vartheta)=0, \\
n_{2} B_{2} \exp (i \psi)+\mathbf{s} \mathbf{H}_{0} \exp (-\vartheta)+\mathbf{s} \mathbf{H}_{0}^{\prime} \exp (\vartheta)=0 .
\end{gathered}
$$

Здесь $\vartheta=\gamma \xi^{\prime}, \psi=\eta_{1} \xi^{\prime}$, причем далее принято $n_{2}=n_{1}$, $\left(\eta_{2}=\eta_{1}\right)$, т. е. окружающие анизотропный слой изотропные среды одинаковы.

Раскрывая входящие в эти уравнения скалярные произведения соответствующих векторов с учетом соотношений (2), (6)-(9), систему уравнений (11) можно преобразовать к следующему виду:

$$
\begin{gathered}
\eta_{1} \xi /(n \gamma)\left(A_{1}-A_{1}^{\prime}\right)=\gamma\left(C_{1}+C_{1}^{\prime}\right)-\delta\left(C_{2}-C_{2}^{\prime}\right), \\
\eta_{1} \xi /(n \gamma) A_{2} \exp (i \psi)=\gamma\left(C_{1 \vartheta}+C_{1 \vartheta}^{\prime}\right) \\
+\delta(\vartheta-1) C_{2 \vartheta}+\delta(\vartheta+1) C_{2 \vartheta}^{\prime}, \\
-\xi\left(A_{1}+A_{1}^{\prime}\right)=i n \gamma\left(C_{1}-C_{1}^{\prime}\right), \\
-\xi A_{2} \exp (i \psi)=i n\left(\gamma\left(C_{1 \vartheta}-C_{1 \vartheta}^{\prime}\right)+\delta \vartheta\left(C_{2 \vartheta}-C_{2 \vartheta}^{\prime}\right)\right), \\
-n_{1} \xi / \varepsilon_{0}\left(B_{1}+B_{1}\right)=-\gamma\left(C_{1}+C_{1}^{\prime}\right)+\left(2 \xi^{2}+\delta\right)\left(C_{2}-C_{2}^{\prime}\right), \\
-n_{1} \xi / \varepsilon_{0} B_{2} \exp (i \psi)=-\gamma\left(C_{1}+C_{1 \vartheta}^{\prime}\right)-\delta \vartheta\left(C_{2 \vartheta}+C_{2 \vartheta}^{\prime}\right) \\
+\left(2 \xi^{2}+\delta\right)\left(C_{2 \vartheta}-C_{2 \vartheta}^{\prime}\right), \\
\eta_{1} / n_{1}\left(B_{1}+B_{1}^{\prime}\right)=i \gamma^{2} / \xi\left(C_{1}-C_{1}^{\prime}\right)-2 i \gamma \xi\left(C_{2}+C_{2}^{\prime}\right), \\
\eta_{1} / n_{1} B_{2} \exp (i \psi)=i \gamma^{2} / \xi\left(C_{1 \vartheta}+C_{1 \vartheta}^{\prime}\right) \\
-2 i \gamma \xi\left(C_{2 \vartheta}+C_{2 \vartheta}^{\prime}\right)+i \gamma \delta \vartheta / \xi\left(C_{2 \vartheta}-C_{2 \vartheta}^{\prime}\right) . \quad(12)
\end{gathered}
$$

Здесь $n=\sqrt{\varepsilon_{o}}, \quad C_{1 \vartheta}=C_{1} \exp (-\vartheta), \quad C_{2 \vartheta}=C_{2} \exp (-\vartheta)$, $C_{1 \vartheta}^{\prime}=C_{1}^{\prime} \exp (\vartheta), C_{2 \vartheta}^{\prime}=C_{2}^{\prime} \exp (\vartheta)$.

Исключая в (12) амплитуды $A_{1}^{\prime}, B_{1}^{\prime}, A_{2}, B_{2}$, получим систему четырех уравнений вида

$$
\begin{gathered}
2 \eta_{1} \xi /(n \gamma) A_{1}+i p^{*} C_{1}-i p C_{1}^{\prime}+\delta\left(C_{2}-C_{2}^{\prime}\right)=0, \\
2 n_{1} \eta_{1} \xi B_{1}-\gamma u C_{1}-\gamma u^{*} C_{1}^{\prime}+\left(\varepsilon_{0} \eta_{1} \delta+2 \xi^{2} u\right) C_{2} \\
-\left(\varepsilon_{0} \eta_{1} \delta+2 \xi^{2} u^{*}\right) C_{2}^{\prime}=0
\end{gathered}
$$

$p C_{1 \vartheta}+i \delta\left(1-i p \xi^{\prime}\right) C_{2 \vartheta}-p^{*} C_{1 \vartheta}^{\prime}-i \delta\left(1-i p^{*} \xi^{\prime}\right) C_{2 \vartheta}^{\prime}=0$,

$$
\begin{gathered}
\gamma u^{*} C_{1 \vartheta}+\gamma u C_{1 \vartheta}^{\prime}-\left[\varepsilon_{0} \eta_{1} \delta+\left(2 \xi^{2}-\delta \vartheta\right) u^{*}\right] C_{2 \vartheta} \\
+\left[\varepsilon_{0} \eta_{1} \delta+\left(2 \xi^{2}+\delta \vartheta\right) u\right] C_{2 \vartheta}^{\prime}=0,
\end{gathered}
$$

где $p=\eta_{1}-i \gamma, u=\varepsilon_{0} \eta_{1}+i \varepsilon_{1} \gamma$.

Таким образом, получена система четырех алгебраических уравнений относительно неизвестных $C_{1}\left(C_{2}\right)$, $C_{1}^{\prime}\left(C_{2}^{\prime}\right)$ (последние входят в соответствующие выражения (6), (8) для $\mathbf{E}_{0}\left(\mathbf{E}_{0}^{\prime}\right)$ и $\left.\mathbf{H}_{0}\left(\mathbf{H}_{0}^{\prime}\right)\right)$.

Заметим, что при $z \rightarrow \infty\left(C_{1}^{\prime}=C_{2}^{\prime}=0\right)$ последние два уравнения выполняются тождественно, а из первых двух следуют выражения для $C_{1}$ и $C_{2}$, совпадающие с соответствующими выражениями для случая отражения от границы раздела с полубесконечным гиперболическим метаматериалом. В этом случае нетрудно получить также выражения для амплитуд $A_{1}^{\prime}$ и $B_{1}^{\prime}$ отраженной волны, которые в конечном счете определяют соответствующие 
амплитудные (и энергетические) коэффициенты отражения. Для них имеют место следующие соотношения:

$A_{1}^{\prime}=1 / \Delta\left\{\left[2 \xi^{2} p u+\delta\left(\varepsilon_{0} \eta_{1}^{2}-\varepsilon_{1} \gamma^{2}\right)\right] A_{1}-2 \eta_{1} \gamma \sqrt{\varepsilon_{o} \varepsilon_{1}} \delta B_{1}\right\}$,

$B_{1}^{\prime}=1 / \Delta\left\{\left[2 \xi^{2} p^{*} u^{*}+\delta\left(\varepsilon_{0} \eta_{1}^{2}-\varepsilon_{1} \gamma^{2}\right)\right] B_{1}+2 \eta_{1} \gamma \sqrt{\varepsilon_{o} \varepsilon_{1}} \delta A_{1}\right\}$,

где $\Delta=\xi^{2}\left[2 p^{*} u+\delta\left(\varepsilon_{0}-\varepsilon_{1}\right)\right]$.

Так как амплитуды отраженной волны являются комплексными величинами, то отраженная волна $\mathbf{E}_{1}^{\prime}$ является эллиптически поляризованной $\left(\left[\mathbf{E}_{1}^{\prime} \mathbf{E}_{1}^{\prime *}\right] \neq 0\right)$. Нетрудно убедиться, что в этом случае энергетический коэффициент отражения $R$, определяемый как отношение $\left|\mathbf{E}_{1}^{\prime}\right|^{2} /\left|\mathbf{E}_{1}\right|^{2}$, равен единице,

$$
R=\frac{\left|\mathbf{E}_{1}^{\prime}\right|^{2}}{\left|\mathbf{E}_{1}\right|^{2}}=\frac{\left|A_{1}^{\prime}\right|^{2}+\left|B_{1}^{\prime}\right|^{2}}{\left|A_{1}\right|^{2}+\left|B_{1}\right|^{2}}=1,
$$

что и доказывает наличие явления полного отражения.

Отметим, что, поскольку амплитуды отраженной волны содержат обе составляющие падающей волны, то общепринятое определение амплитудных коэффициентов отражения для изотропных сред $r_{s}=\left|A_{1}^{\prime}\right| /\left|A_{1}\right|$ и $r_{p}=\left|B_{1}^{\prime}\right| /\left|B_{1}\right|$ здесь, очевидно, неприемлемо вследствие невыполнимости условия равенства единице соответствующих энергетических коэффициентов, т.е. $R_{s}=\left|r_{s}\right|^{2} \neq 1$ и $R_{p}=\left|r_{p}\right|^{2} \neq 1$, что не должно быть в случае полного отражения. Поэтому в данном случае амплитудные коэффициенты отражения можно корректно ввести (имея в виду соотношение (15)) следующим образом: $r_{s}=\left|A_{1}^{\prime}\right| /\left|\mathbf{E}_{1}\right|$ и $r_{p}=\left|B_{1}^{\prime}\right| /\left|\mathbf{E}_{1}\right|$. При таком определении энергетический коэффициент отражения для случая полубесконечной анизотропной среды $R=\left|r_{s}\right|^{2}+\left|r_{p}\right|^{2}=1$.

Для случая, когда углы падения $\alpha$ близки к предельному углу полного отражения $\left(\sin \alpha_{0}=\sqrt{\varepsilon_{0} / \varepsilon_{1}}\right)$, а толщина слоя удовлетворяет условию $h / \lambda \leq 1$, т. е. когда величины $\xi^{\prime}=k h$ и $\vartheta=\gamma \xi^{\prime}$ весьма малы, из (12) можно получить, что при изменении поляризации падающей волны энергетический коэффициент отражения изменяется от максимального значения $R_{\max }$ до минимального $R_{\min }$, которые при $\vartheta \ll 1$ пропорциональны $\vartheta^{2}$ и удовлетворяют уравнениям

$$
\begin{aligned}
\Sigma=R_{\max }+R_{\min }= & \vartheta^{2}\left((v+1 / v)^{2}+(\mu+1 / \mu)^{2}\right. \\
& \left.+4 \beta\left(v^{2}-\mu^{2}\right)+4 \beta^{2}(v+\mu)^{2}\right) / 4 \\
\Pi=R_{\max } R_{\min }= & \vartheta^{4}((\mu+1 / \mu)(v+1 / v) \\
& +2 \beta(v / \mu-\mu / v))^{2} / 16
\end{aligned}
$$

где $\mu=\gamma / \eta_{1}, v=\varepsilon_{0} \eta_{1} /\left(\varepsilon_{1} \gamma\right), \beta=\delta / 2 \xi^{2}$. Из (16) получаем, что

$$
R_{\max }, R_{\min }=\Sigma / 2 \pm \sqrt{\Sigma^{2} / 4-\Pi}
$$

В отсутствие анизотропии $(\delta=\beta=0)$ величины $R_{\max }, R_{\min }$ равны энергетическим коэффициентам отражения для тонких слоев при поляризациях, перпендикулярной и параллельной плоскости падения, $R_{s}=\vartheta^{2}(\mu+1 / \mu)^{2} / 4, R_{p}=\vartheta^{2}(v+1 / v)^{2} / 4$.
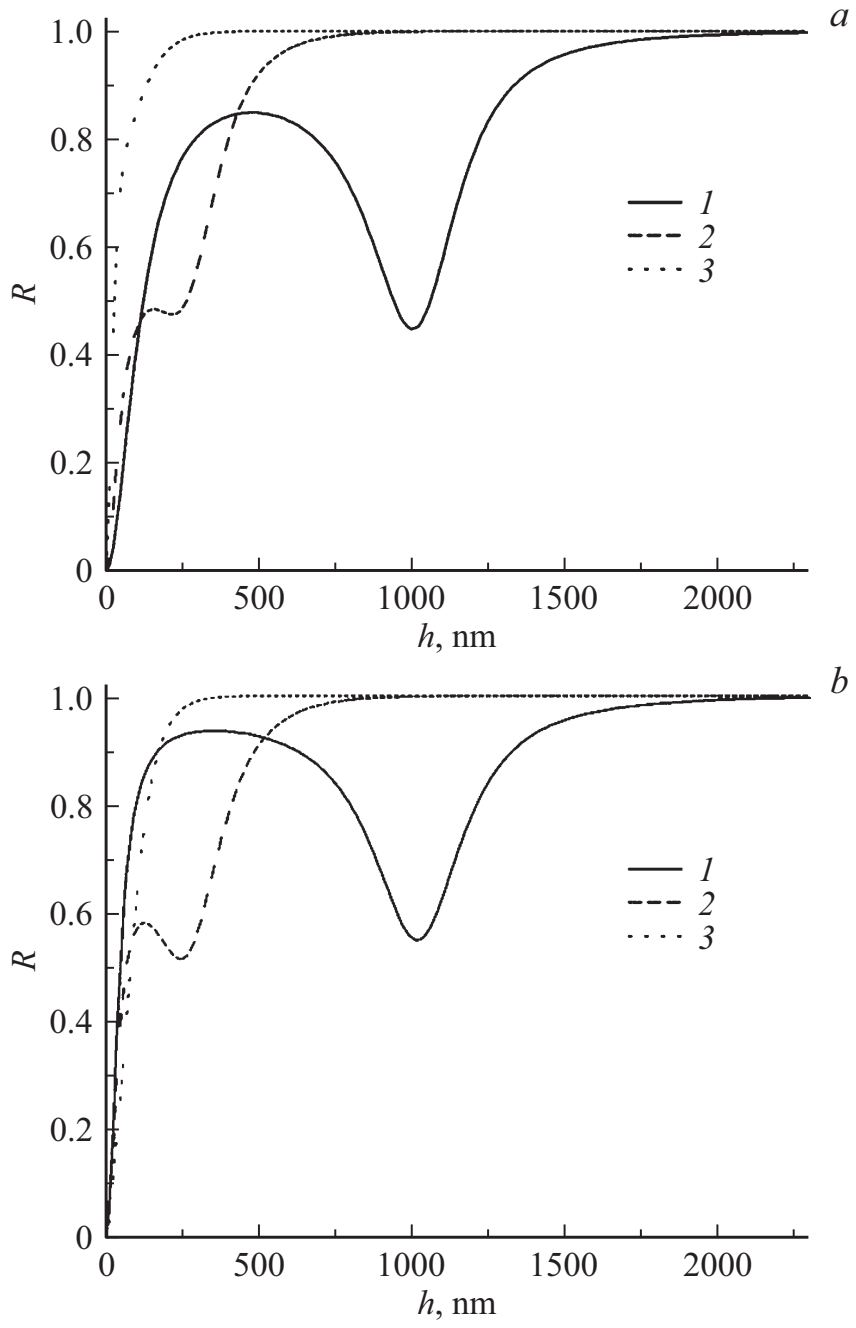

Рис. 2. Зависимость коэффициента отражения плоской электромагнитной волны от толщины слоя гиперболического метаматериала на основе слоисто-периодической металлодиэлектрической наноструктуры ITO/Ag, расположенной между двумя полубесконечными стеклами ВК7, при распространении в слое особой неоднородной волны. Угол падения света на структуру $\alpha$ и угол между плоскостью падения и главной плоскостью ГММ $\rho$ составляют соответственно $51.55^{\circ}$ и $4.58^{\circ}(1), 53.63^{\circ}$ и $14.2^{\circ}(2), 63.77^{\circ}$ и $29.51^{\circ}$ (3). Длина волны света $360 \mathrm{~nm}$, диэлектрическая проницаемость стекла $\varepsilon_{1}=2.363$. Предельный угол полного отражения $\alpha_{0}=51.32^{\circ}$.

\section{Результаты расчетов коэффициента отражения от слоя гиперболического метаматериала при распространении в нем особой неоднородной волны}

Рассмотрим слой, образованный слоисто-периодической металлодиэлектрической структурой. В приближении эффективной среды, когда толщина каждого входящего в структуру слоя достаточно мала, т.е. $\left|k_{d} d_{d}\right| \ll 1,\left|k_{m} d_{m}\right| \ll 1$, где $k_{d}, k_{m}$ - соответственно волновые числа диэлектрического и металлического слоев, 
многослойная структура может рассматриваться как эффективная оптически одноосная среда. При этом тензор диэлектрической проницаемости ее имеет собственные значения, определяемые выражениями

$$
\varepsilon_{o}=(1-f) \varepsilon_{d}+f \varepsilon_{m}, \quad \varepsilon_{e}=\left[\frac{1-f}{\varepsilon_{d}}+\frac{f}{\varepsilon_{m}}\right]^{-1} .
$$

Здесь $f=d_{m} /\left(d_{m}+d_{d}\right)$ - фактор заполнения (объемная доля, занимаемая в структуре металлом), $\varepsilon_{d}, \varepsilon_{m}-$ соответственно проницаемости диэлектрического и металлического слоев. При этом величина $\varepsilon_{m}$ описывается формулой Друде [11]:

$$
\begin{aligned}
\varepsilon_{m}(\omega) & =\varepsilon_{\infty}-\omega_{p}^{2} /\left(\omega^{2}+i \omega \Gamma\right) \\
& =\varepsilon_{\infty}-\omega_{p}^{2} /\left(\omega^{2}+\Gamma^{2}\right)+i \omega_{p}^{2} \Gamma /\left[\omega\left(\omega^{2}+\Gamma^{2}\right)\right],
\end{aligned}
$$

где $\omega_{p}-$ плазмонная частота, $\varepsilon_{\infty}-$ постоянная, описывающая вклад межзонных переходов, $\Gamma=V_{F} / l-$ постоянная затухания, $V_{F}-$ скорость Ферми, $l-$ среднее значение свободного пути электрона в объемном металле. Для серебра, например, $\varepsilon_{\infty}=5, \omega_{p}=14 \cdot 10^{15} \mathrm{~s}^{-1}$, $\Gamma=32 \cdot 10^{12} \mathrm{~s}^{-1}, V_{F}=1.4 \cdot 10^{6} \mathrm{~ms}^{-1}[1]$. Расчет, согласно выражения (18) для слоисто-периодической среды ITO/Ag $\left(d_{m}=20 \mathrm{~nm}, f=0.3\right)$ при длине падающей световой волны $360 \mathrm{~nm}$, дает значения $\varepsilon_{o}=1.44$, $\varepsilon_{e}=-6.32$. Для граничащих со слоем изотропных сред (оптическое стекло ВК7) $\varepsilon_{1}=n_{1}^{2}=2.36$.

Нами выполнен расчет коэффициента отражения слоя путем численного решения уравнений (12). На рис. 2 представлена зависимость коэффициента отражения $s$ - и $p$-поляризованной волны от толщины слоя. Как видно из рис. 2, при малых толщинах слоя $(<50 \mathrm{~nm})$ коэффициент отражения от него невелик, что объясняется явлением „просачивания“ энергии. При увеличении толщины $h$ коэффициент отражения возрастает до единицы, что соответствует случаю полного внутреннего отражения. Однако наличие в слое неоднородной волны особого типа приводит к изменению $R$ и возникновению в зависимости $R(h)$ минимума $R_{\min }$, проявляющегося при приближении угла падения к предельному $\alpha_{0}$ (рис. 2). $R_{\min }$ оказывается зависящим от состояния поляризации падающего света. Так, например, для угла падения света $51.55^{\circ}\left(\alpha_{0}=51.32^{\circ}\right)$ и угла между плоскостью падения и главной плоскостью металлодиэлектрической структуры $4.58^{\circ}$ для случая $h=1 \mu \mathrm{m} R_{\min }=0.45$ в случае $s$-поляризованных волн и $R_{\min }=0.55$ для $p$-поляризованных волн.

\section{Заключение}

Таким образом, в настоящей работе исследованы особенности отражения света от слоя гиперболического метаматериала в условиях распространения в нем волны особого типа, амплитуда которой убывает внутри анизотропной среды при удалении от ее поверхности по сложному неэкспоненциальному закону. Выполнены численные расчеты коэффициента отражения от слоя наноструктуры ITO/Ag в условиях распространения в ней особых неоднородных волн. Показано, что формируемая в слое неоднородная волна, характеризуемая неэкспоненциальным уменьшением продольного энергетического потока, обусловливает появление минимума в зависимости коэффициента отражения от толщины слоя и, следовательно, возникновение явления „просачивания“ энергии при соответствующей толщине. При этом данный эффект оказывается зависящим от состояния поляризации падающего света: а именно для $s$ поляризованных волн падение коэффициента отражения более выражено. Отметим, что этот эффект наблюдается при достаточно больших толщинах слоя (в рассмотренном случае $h / \lambda \sim 2.7)$.

Полученные результаты имеют перспективы применения в микроскопии, а также при разработке новых методов зондирования приповерхностных дефектов различных материалов.

\section{Список литературы}

[1] Cai $W$., Shalaev V. Optical Metamaterials: Fundamentals and Applications. Springer, 2010. doi 10.1007/978-1-4419-1151-3

[2] Metamaterials Handbook 1: Theory and Phenomena of Metamaterials. CRC Press, 2009.

[3] Pendry J.B. // Phys. Rev. Lett. 2000. V. 85. P. 3966. doi 10.1103/PhysRevLett.85.3966

[4] Fang N., Lee H., Sun C., Zhang X. // Science. 2005. V. 308. P. 534. doi 10.1126/science.1108759

[5] Kidwai O., Zhukovsky S.V., Sipe J.E. // Phys. Rev. 2012. V. A 85. P. 053842. doi 10.1103/PhysRevA.85.053842

[6] Shekhar P., Atkinson J., Jacob Z. // Nano Convergence. 2014. V. 1. P. 14. doi 10.1186/s40580-014-0014-6.

[7] Федоров Ф.И. Теория гиротропии. Минск, 1976.

[8] Федоров Ф.И., Петров Н.С. // Опт. и спектр. 1963. Т. 14. B. 2. C. 256.

[9] Kurilkina S.N., Petrov N.S., Zimin A.B., Belyi V.N. // J. Opt. 2017. V. 19. P. 125102. doi 10.1088/2040-8986/aa945c

[10] Федоров Ф.И. Оптика анизотропных сред. Минск: АНБССР, 1958. $381 \mathrm{c}$.

[11] Kurilkina S.N., Binhussain M.A., Belyi V.N., Kazak N.S. // J. Opt. 2016. V. 18. P. 085102. doi 10.1088/2040$8978 / 18 / 8 / 085102$ 